\title{
Lift-off invariant inductance of steels in multi-frequency eddy-current testing
}

\author{
Mingyang $\mathrm{Lu}^{\mathrm{a}, 1, *}$, Xiaobai Menga ${ }^{\mathrm{a}, \mathrm{b}, 1}$, Ruochen Huang ${ }^{\mathrm{a}}$, Liming Chen ${ }^{\mathrm{a}}$, Anthony Peyton ${ }^{\mathrm{a}}$, Wuliang Yin ${ }^{\mathrm{a}, *}$ \\ ${ }^{\text {a }}$ School of Electrical and Electronic Engineering, University of Manchester, Oxford Road, Manchester, M13 9PL, UK \\ ${ }^{\mathrm{b}}$ Faculty of Art, Science and Technology, University of Northampton, Northampton, NN1 5PH, UK \\ ${ }^{1} \mathrm{M}$. Lu and X. Meng contributed equally and share the first authorship. \\ *Corresponding author: mingyang.lu@manchester.ac.uk; wuliang.yin@manchester.ac.uk
}

\begin{abstract}
Eddy current testing can be used to interrogate steels but it is hampered by the lift-off distance of the sensor. Previously, the lift-off point of intersection (LOI) feature has been found for the pulsed eddy current (PEC) testing. In this paper, a lift-off invariant inductance (LII) feature is proposed for the multi-frequency eddy current (MEC) testing, which merely targets the ferromagnetic steels. That is, at a certain working frequency, the measured inductance signal is found nearly immune to the lift-off distance of the sensor. Such working frequency and inductance are termed as the lift-off invariant frequency (LIF) and LII. Through simulations and experimental measurements of different steels under the multi-frequency manner, the LII has been verified to be merely related to the sensor parameters and independent of different steels. By referring to the LIF of the test piece and using an iterative inverse solver, one of the steel properties (either the electrical conductivity or magnetic permeability) can be reconstructed with a high accuracy.
\end{abstract}

Index Terms - Eddy current testing; lift-off invariance; property measurement; multi-frequency; non-destructive testing.

\section{Introduction}

Non-destructive testing (NDT) is a commonly used measurement technique for evaluating the property of the material without causing damage. As one of the typical NDT techniques, the eddy current (EC) testing is widely applied for the measurement of sample properties and defect inspection owing to its rich information, high adaptability and sensitivity [1-9]. By referring to the signals from the transmitters and receivers of EC sensors, the electromagnetic characteristic of the test piece can be derived in a contactless manner [10-16]. Currently, the lift-off distance between the EC sensor and sample is one of the major issues in the practical measurement.

Previously, many techniques have been proposed to address the error caused by the lift-off variation when using the multi-frequency eddy current (MEC) testing, pulsed eddy current (PEC) testing, and single-frequency eddy current (SEC) testing [17-27]. The point of intersection (LOI) feature has been proposed by Giguère et al. for the PEC testing [20], which has been further utilised by various researchers to measure the property of the conductive films, multi-layer metallic plates, and steels [21-24]. Tian and Sophian used the normalised method with dual signals to further limit the lift-off effect when using PEC sensors [25]. However, these methods are all suitable for PEC testing. Yin et al. have proposed the peak frequency and zero-crossing frequency features of the MEC spectrum to reconstruct the thickness of non-magnetic material and ferrite fractions of magnetic alloys [26, 27]. However, the reconstruction error was around $8 \%$, which still needs to be further mitigated.

Referring to our previous work, the conductivity invariance phenomenon (CIP) and permeability invariance phenomenon (PIP) have been proposed to tackle the correlation between the electrical conductivity and magnetic permeability at an optimal lift-off distance [28, 29]. Besides, the revised (via the lift-off compensation algorithm) peak frequency and zero-crossing frequency features have been found and used to measure the property of both non-magnetic and magnetic materials [30, 31]. Moreover, different sensor designs (e.g., triple-coil sensor for SEC testing) and measurement techniques (e.g., phase feature for MEC testing) have been used to address the lift-off issue [32-38]. However, the proposed scenarios on reducing measurement error due to the lift-off variation can still be improved.

A lift-off invariant inductance (LII) feature is presented in this paper. It is found that at a certain working frequency, the inductance measured by the eddy current sensor is not affected by the lift-off variation. Such frequency and corresponding inductance are termed as the lift-off invariant frequency (LIF) and lift-off invariant inductance (LII) features. The proposed method only works for ferromagnetic samples. For the austenitic (nonferromagnetic) materials, the inductance change (ideally starts from zero under extremely low-frequency excitation) is negative for different frequencies. Besides, there is no intersected point for inductance change (due to non-ferromagnetic materials) curves at different lift-offs. Experiments on the inductance measurement of three types of steels, including dual-phase 600, low-carbon mild, and Cr-Mo steels have been carried out under the multi-frequency (or swept frequency) mode. The LII feature is found only determined by the geometry of the 
sensor and nearly immune to the test piece. Moreover, the properties (including the magnetic permeability and electrical conductivity) of different steels have been estimated starting by the LIF feature, with a maximum error of $0.61 \%$. The reconstruction is achieved by inputting the known parameters, LIF, and LII into the inverse solver based on the modified Newton Raphson iterative method.

\section{Methodology - Lift-off invariant inductance}

The eddy current sensor can be utilised to test the steel via the measured signal, including the impedance and inductance. However, the lift-off the sensor distance can significantly affect the detected signals. To address the lift-off issue of the sensor, features of lift-off invariant inductance (LII) and lift-off invariant frequency (LIF) have been proposed. Then the properties of the steels can be reconstructed by referring to LIF using an iterative inverse solver (modified Newton-Raphson method).

\section{A. Forward solver - analytical formulation of inductance change}

To initiate the investigation of the LII and LIF features, the analytical formulation of the inductance for two circular transmitting/receiving coils (with one transmitter and receiver) co-axially deployed above the half-space material is introduced [39].

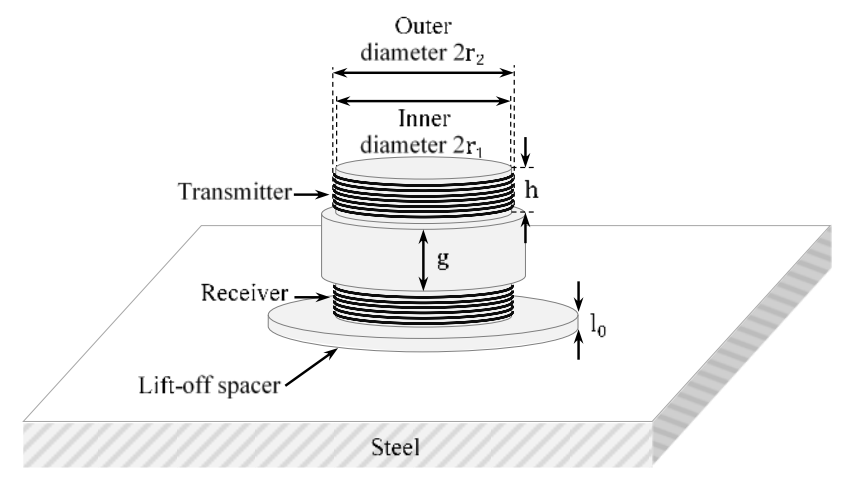

Fig. 1. Eddy current sensor with two circular coils co-axially wound is parallel to the steel plate with a certain lift-off

As illustrated in Fig. 1, the air-core sensor is composed of one transmitter and one receiver. Two circular transmitting/receiving coils with the identical spiral height $h$, inner radius $r_{1}$ and outer radius $r_{2}$ are co-axially wound on the ceramic rod with a separation distance of $g$. The eddy current sensor is placed parallel to the steel plate with a small lift-off of $l_{0}$. It was found that the induced eddy current would not be restrained by the edge of the steel when the plate planar size is around 5 times larger than the diameter of the transmitting/receiving coil. Consequently, the steel plate can be treated as an infinite half-space material. Besides, the change of mutual inductance on the sensor due to the steel is defined as

$$
\begin{gathered}
\mathrm{L}=\mathrm{K} \int_{0}^{\infty} \mathrm{M}(\alpha) \phi(\alpha) \mathrm{d} \alpha \\
\mathrm{K}=\frac{\pi \mu_{0} \mathrm{~N}^{2}\left(\mathrm{r}_{1}+\mathrm{r}_{2}\right)}{2 \mathrm{~h}^{2}\left(\mathrm{r}_{2}-\mathrm{r}_{1}\right)^{2}}
\end{gathered}
$$

There exists a restriction for the radius of the transmitting/receiving coils. That is, the planar dimension of the test piece should be at least 5 times of the radius of the transmitting/receiving coils [28]. Otherwise, the induced eddy current will be confined by the cutting edge of the steel plate, and the fundamental formula (equation 1) will be invalid. In (2), $N$ is the turns of the transmitter and receiver. $\mu_{0}$ is the magnetic permeability of the free space. In (1), $M(\alpha)$ is determined by the geometry of the sensor and defined in (3).

$$
M(\alpha)=\frac{P^{2}(\alpha)}{\alpha^{6}} \mathrm{e}^{-\alpha\left(2 \mathrm{l}_{0}+\mathrm{g}+\mathrm{h}\right)}\left(\mathrm{e}^{-\alpha \mathrm{h}}-1\right)^{2}
$$

$\phi(\alpha)$ in (1) is determined by the property of the steel, which is defined as

$$
\begin{gathered}
\phi(\alpha)=\operatorname{Re}\left(\frac{\left(\alpha_{1}+\mu_{1} \alpha\right)\left(\alpha_{1}-\mu_{1} \alpha\right)-\left(\alpha_{1}+\mu_{1} \alpha\right)\left(\alpha_{1}-\mu_{1} \alpha\right) \mathrm{e}^{2 \alpha_{1} c}}{-\left(\alpha_{1}-\mu_{1} \alpha\right)\left(\alpha_{1}-\mu_{1} \alpha\right)+\left(\alpha_{1}+\mu_{1} \alpha\right)\left(\alpha_{1}+\mu_{1} \alpha\right) \mathrm{e}^{2 \alpha_{1} c}}\right) \\
\alpha_{1}=\sqrt{\alpha^{2}+j 2 \pi f \sigma \mu_{1} \mu_{0}}
\end{gathered}
$$


In (5), $\mathrm{f}$ is the working frequency. $\sigma$ is the electrical conductivity of the steel. $\mu_{1}$ is the relative magnetic permeability of the sample. Moreover, $\mathrm{P}(\alpha)$ in (3) is defined in (6), which is related to the first-order Bessel function of the first kind $\mathrm{J}_{1}$.

$$
P(\alpha)=\int_{\alpha \mathrm{r}_{1}}^{\alpha \mathrm{r}_{2}} \rho \mathrm{J}_{1}(\rho) \mathrm{d} \rho
$$

\section{B. Lift-off invariant inductance and frequency - LII and LIF}

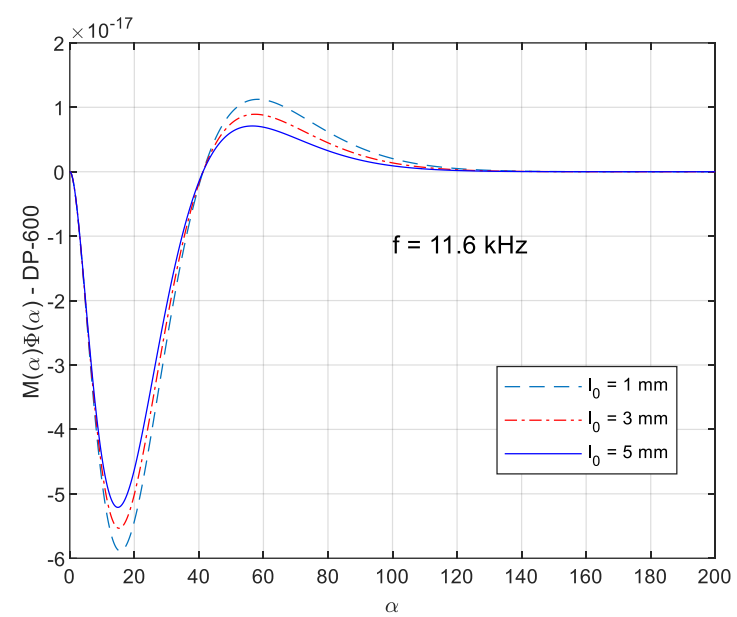

(a)

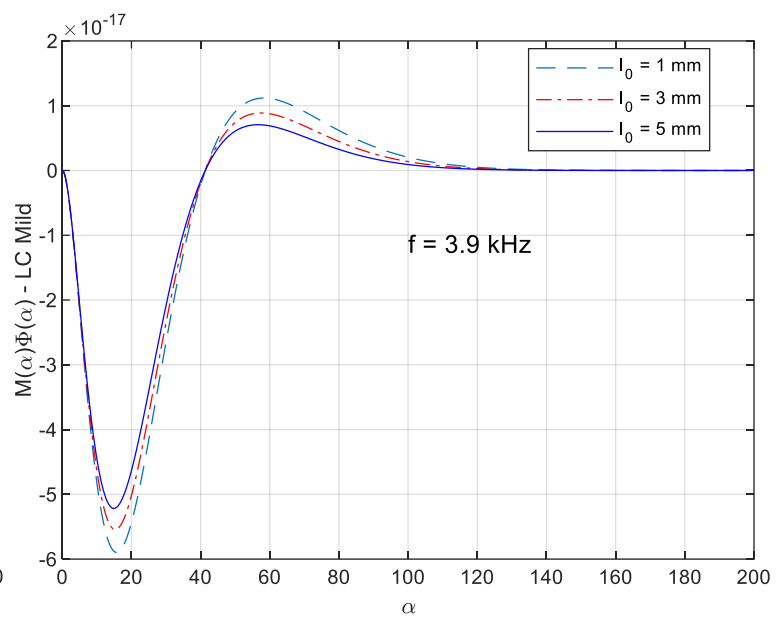

(b)

Fig. $2 \mathrm{M}(\alpha) \phi(\alpha)$ versus $\alpha$ for: (a) DP-600 at $11.60 \mathrm{kHz}$ (b) Low-carbon Mild steel at $3.90 \mathrm{kHz}$

The electrical and magnetic properties of the samples are reported in Table1 while the characteristics of the sensor are summarized in Table 2

From multiple trials of inductance (change) calculations with different sample properties and working frequencies, it has been found the inductance change $\mathrm{L}$ is almost immune to the lift-off variations under a certain working frequency. For example, Fig. 2 exhibits the integrand in (1) - $M(\alpha) \phi(\alpha)$ for the ferrite-austenite dualphase (DP) 600 and low-carbon (LC) mild steels (Table 1) with a series of lift-offs. The parameters of coils in $\mathrm{M}(\alpha)$ can be referred to Table 2. It can be observed that, under the working frequency of $11.6 \mathrm{kHz}$ for DP-600 and $3.9 \mathrm{kHz}$ for LC mild steel, the curves of the integrand - $\mathrm{M}(\alpha) \phi(\alpha)$ with different lift-offs are nearly the same. Moreover, the integrated inductance change (integral in (1)) for both curves has an identical value $\mathrm{L}_{0}=$ $-1.195 \mathrm{nH}$. As can be observed from the red dot-dash line in Fig. 3, the inductance value $\mathrm{L}_{0}$ is almost immune to the lift-off variations. The inductance value $\mathrm{L}_{0}$ is constant and can be termed as the lift-off invariant inductance (LII). The LII $-\mathrm{L}_{0}$ is found to be independent of the test piece and merely determined by the geometry of the sensor. Besides, the corresponding frequency when the inductance change approaches LII - $\mathrm{L}_{0}$ is termed as the lift-off invariant frequency (LIF).

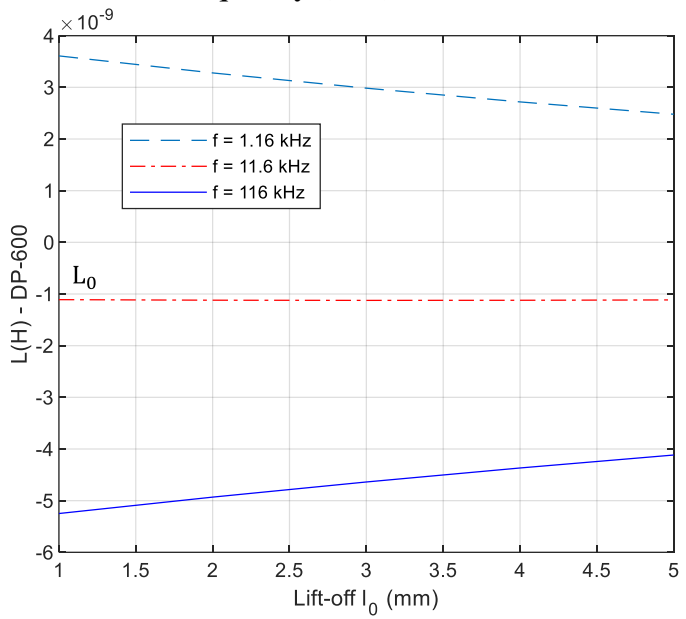

(a)

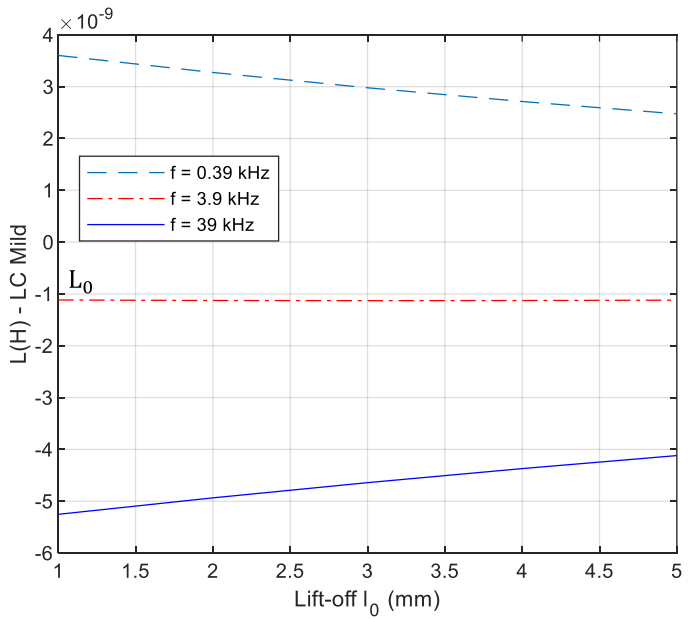

(b)

Fig. 3 Inductance change - L for the sensor in Table 2 with different lift-offs

(a) analytical result of DP-600 steel under working frequencies of $1.16,11.60$, and $116.00 \mathrm{kHz}$

(b) analytical result of Low-carbon Mild steel under working frequencies of $0.39,3.90$, and $39.00 \mathrm{kHz}$ 


\section{Inverse solver - iterative method}

Since the inductance change in the presence of different samples achieves the LII - $\mathrm{L}_{0}$ value at different frequency values. The properties of the steel can be reconstructed by finding the corresponding frequency - LIF at which $\mathrm{L}=\mathrm{L}_{0}$ under the multi-frequency excitation, by choosing the frequency for which $\mathrm{L}$ is the closest to $\mathrm{L}_{0}$. Therefore, one of the steel properties (either the electrical conductivity or the relative magnetic permeability; one must be known as a priori) can be reconstructed by finding the minimum value of $\Delta \mathrm{L}$ when varying $\sigma$ or $\mu_{1}$.

$$
\Delta \mathrm{L}\left(\mu_{1}, \sigma\right)=\left|\mathrm{L}\left(\mu_{1}, \sigma\right)-\mathrm{L}_{0}\right|
$$

In (7), $L\left(\mu_{1}, \sigma\right)$ is the analytical inductance change using (1) when inputting $\mu_{1}, \sigma$, and the corresponding frequency - LIF (refer to the measured multi-frequency inductance change) at or closest to LII - $\mathrm{L}_{0}$.

One of the efficient inverse solvers for retrieving $\sigma$ or $\mu_{1}$ is the modified Newton-Raphson iterative method, which can determine increasing or decreasing reference values of $\mu_{1}$ or $\sigma$ when finding the minimum $\Delta \mathrm{L}$ [34].

$$
\begin{gathered}
\mu_{1}=\mu_{1 \mathrm{r}}-\mathrm{J}_{\mu}^{-1}\left(\mathrm{~L}\left(\mu_{1 \mathrm{r}}\right)-\mathrm{L}_{0}\right) \\
\sigma=\sigma_{\mathrm{r}}-\mathrm{J}_{\sigma}^{-1}\left(\mathrm{~L}\left(\sigma_{\mathrm{r}}\right)-\mathrm{L}_{0}\right)
\end{gathered}
$$

In (8) and (9), $\mu_{1 \mathrm{r}}$ and $\sigma_{\mathrm{r}}$ are the reference values under the current iterative loop. 6 different initial reference values (for the first iterative loop) have been used for the retrieval of electrical conductivity or relative magnetic permeability. Each initial reference value is used in an independent parallel iterative solver for searching the local minimum convergence threshold (maximum interference threshold (MAXIT)) / residual of the inductance. That is, values of $1 \mathrm{MS} / \mathrm{m}, 10 \mathrm{MS} / \mathrm{m}, 20 \mathrm{MS} / \mathrm{m}, 30 \mathrm{MS} / \mathrm{m}, 40 \mathrm{MS} / \mathrm{m}, 50 \mathrm{MS} / \mathrm{m}$ are used for the retrieval of electrical conductivity; and values of 50,100, 200,300,400, 500 are used for the retrieval of relative magnetic permeability. And the global solution of the retrieval is by finding the local solution with the smallest residual value (of the inductance). These 6 different initial values are randomly set. In practice, a crude test of the conductivity (using the four-terminal method) or permeability fitting (using air-core or ferrite-core sensors) will hasten the convergence.

For the input of multiple frequencies, $J_{\mu}$ and $J_{\sigma}$ are the Jacobian matrix. For the (single) corresponding frequency - LIF at or closest to LII - L_ $0, J_{\mu}$ and $J_{\sigma}$ are single values and defined in (10) and (11).

$$
\begin{gathered}
J_{\mu}=\frac{L\left(\mu_{1 \mathrm{r}}\right)-L\left(\mu_{1 \mathrm{r}}^{\prime}\right)}{\mu_{1 \mathrm{r}}-\mu_{1 \mathrm{r}}^{\prime}} \\
\mathrm{J}_{\sigma}=\frac{\mathrm{L}\left(\sigma_{\mathrm{r}}\right)-\mathrm{L}\left(\sigma_{\mathrm{r}}^{\prime}\right)}{\sigma_{\mathrm{r}}-\sigma_{\mathrm{r}}^{\prime}}
\end{gathered}
$$

In (10) and (11), $\mu_{1 \mathrm{r}}^{\prime}$ and $\sigma_{\mathrm{r}}^{\prime}$ are the values approaching $\mu_{1 \mathrm{r}}$ and $\sigma_{\mathrm{r}}$, which can be defined as $0.99 \mu_{1 \mathrm{r}}$ and $0.99 \sigma_{\mathrm{r}} . \mathrm{L}\left(\mu_{1 \mathrm{r}}^{\prime}\right)$ and $\mathrm{L}\left(\sigma_{\mathrm{r}}^{\prime}\right)$ are the inductance change when inputting $\mu_{1 \mathrm{r}}^{\prime}, \sigma_{\mathrm{r}}^{\prime}$, and the corresponding frequency LIF at or closest to LII - $\mathrm{L}_{0}$ into forward analytical formulation - equation (1). The iterative loop can be terminated when $\left(\mathrm{L}\left(\mu_{1 \mathrm{r}}\right)-\mathrm{L}\left(\mu_{1 \mathrm{r}}^{\prime}\right)\right)$ or $\left(\mathrm{L}\left(\sigma_{\mathrm{r}}\right)-\mathrm{L}\left(\sigma_{\mathrm{r}}^{\prime}\right)\right)$ converges at a small maximum interference threshold (MAXIT).

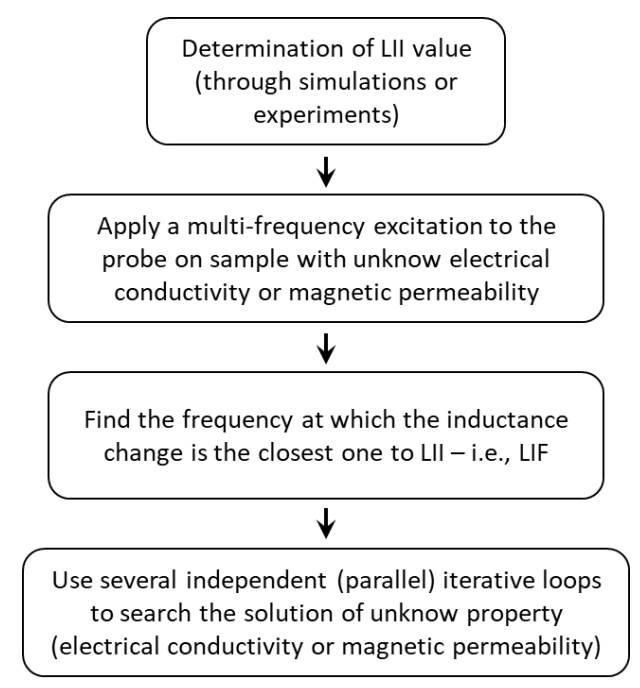

Fig. 4 (a) Flow-chart on the retrieval of the electrical conductivity or magnetic permeability 


\section{Experiments on different steels}

To verify the lift-off invariant inductance (LII) feature, experiments on the multi-frequency (or swept frequency) inductance change of the eddy current sensor above different steels have been carried out. As listed in Table 1, the test pieces selected were three types of steels (including the ferrite-austenite dual-phase 600 alloy, low-carbon mild steel, and Cr-Mo steel) with different electrical conductivities, magnetic permeabilities, and thicknesses. The planar size of these samples $(500 \times 500 \mathrm{~mm})$ is larger than 10 times of the diameter of the sensor. Therefore, the sample plates can be treated as the infinite half-space material and suitable for the analytical forward solver in (1). The parameters of the eddy current sensor in Fig. 1 are listed in Table 2.

Table 1 Properties and thicknesses of steels

\begin{tabular}{|c|c|c|c|}
\hline & DP 600 & $\begin{array}{l}\text { Low-carbon } \\
\text { Mild steel }\end{array}$ & Cr-Mo steel \\
\hline $\begin{array}{l}\text { Electrical conductivity } \\
(\mathrm{MS} / \mathrm{m})\end{array}$ & 4.13 & 8.55 & 3.37 \\
\hline $\begin{array}{l}\text { Relative magnetic } \\
\text { permeability }\end{array}$ & 222 & 154 & 88 \\
\hline Thickness (mm) & 2.4 & 3.0 & 3.0 \\
\hline \multicolumn{4}{|c|}{ Table 2 Parameters of eddy current Sensor } \\
\hline \multicolumn{2}{|c|}{ Turns - N } & 25 & \\
\hline \multicolumn{2}{|c|}{ Coil height $-\mathrm{h} / \mathrm{mm}$} & 12.5 & \\
\hline \multicolumn{2}{|c|}{ Gap - g/mm } & 45.0 & \\
\hline \multicolumn{2}{|c|}{ Inner radius $-r_{1} / \mathrm{mm}$} & 19.5 & \\
\hline \multicolumn{2}{|c|}{ Outer radius $-\mathrm{r}_{2} / \mathrm{mm}$} & 20.0 & \\
\hline \multicolumn{2}{|c|}{ Lift-off $-\mathrm{l}_{0} / \mathrm{mm}$} & 1.0:1.0:5.0 & \\
\hline
\end{tabular}

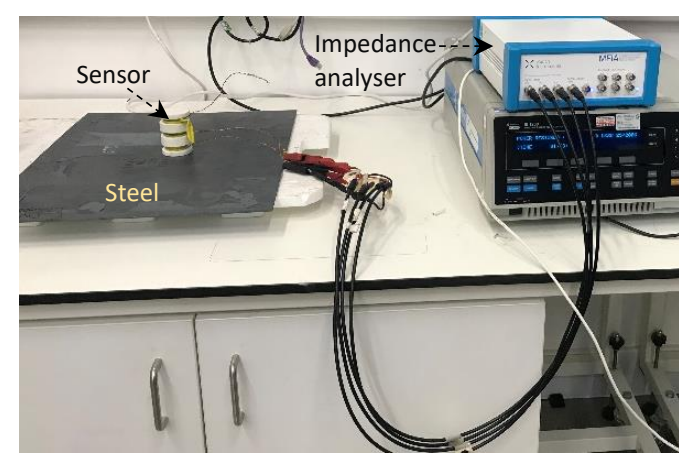

Fig. 4 (b) Eddy current sensor deployed above the sample plate and (top and bottom coils) connected to the impedance analyser

In Fig. 4 (b), the eddy current sensor is placed above the steel. The measurement is conducted under a small range of lift-offs (from 1.0 to $5.0 \mathrm{~mm}$ with an increment of $1.0 \mathrm{~mm}$ ). Two transmitting/receiving coils (top and bottom slot of the sensor in Fig. 4 b), with one transmitter on the top and one receiver at the bottom, are connected to the MFIA impedance analyser. The working frequencies range from $100 \mathrm{~Hz}$ to $500 \mathrm{kHz}$.

\section{Results and discussions}




\section{A. Intersection points of inductance change curves with different lift-offs}

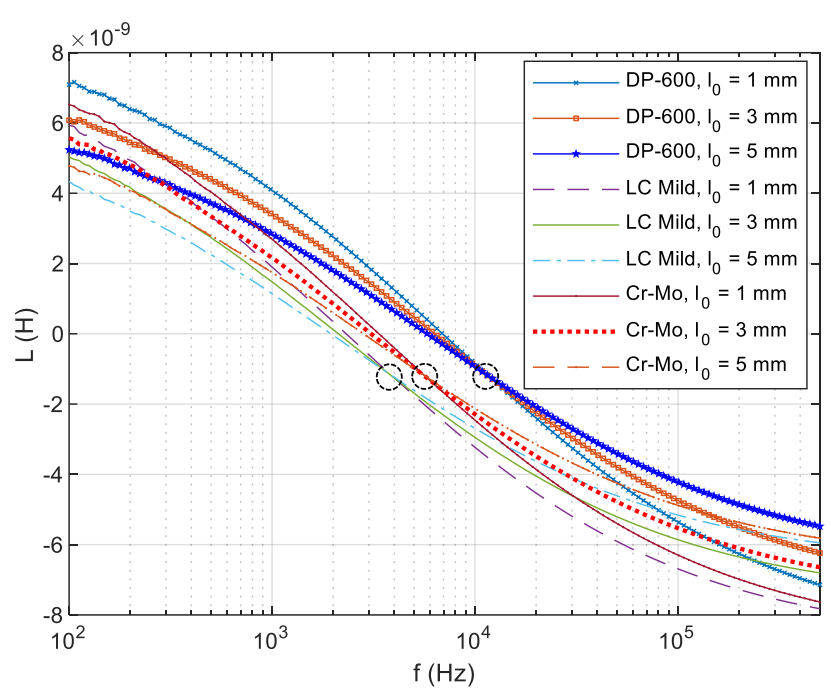

(a)

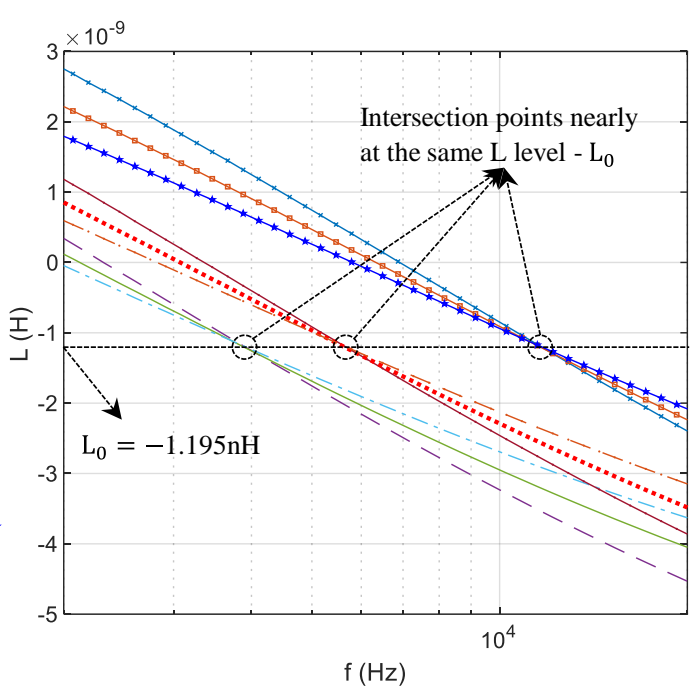

(b)

Fig. 5 Multi-frequency inductance change of the sensor above steels with the lift-off of 1, 3, and $5 \mathrm{~mm}$ (a) full frequency ranges from 0.1 to $500 \mathrm{kHz}$

(b) zooming in frequency range from 2 to $20 \mathrm{kHz}$

Fig. 5 shows the measurement of the multi-frequency inductance change for the eddy current sensor above the steels with lift-off distance of 1,3 , and $5 \mathrm{~mm}$. Several slightly fluctuating points exist at low frequencies (particularly for frequencies lower than $300 \mathrm{~Hz}$ ), which is caused by the low signal-to-noise ratio (SNR) of the impedance analyser when working at low frequencies. It can be observed that the inductance change curves for one sample with different lift-offs cross zero and intersect at a single locus point under a certain frequency. This intersection phenomenon occurs in the ferromagnetic materials (and disappears in austenitic materials). As induced eddy currents reduce inductive energy storage in the sensor, there exists a "zero-crossing" point for the inductance change where the system stores the same energy as it is in the free space [30]. Moreover, the inductance change is shown to be less affected by the lift-off variation under medium frequencies, and almost immune to the lift-off distance under the corresponding frequency - lift-off invariant frequency (LIF) of the overlapped points. Moreover, the LIF is determined by the properties of the sample, including the electrical conductivity, magnetic permeability, and thickness of the steel. As can be seen from the zooming of the inductance change curves in Fig. 5 (b), intersected points for curves of different lift-offs locate at different working frequencies but almost the same inductance change level $\mathrm{L}_{0}=-1.195 \mathrm{nH}$, which is exactly the same as the analytical result of the lift-off invariant inductance (LII) in Fig. 3. The working frequencies closest to the corresponding frequency of the intersected points - LIF for DP-600, low-carbon mild, and Cr-Mo steels are 11.602, 3.889, and $5.637 \mathrm{kHz}$ respectively.

\section{B. Lift-off invariant inductance (LII)}

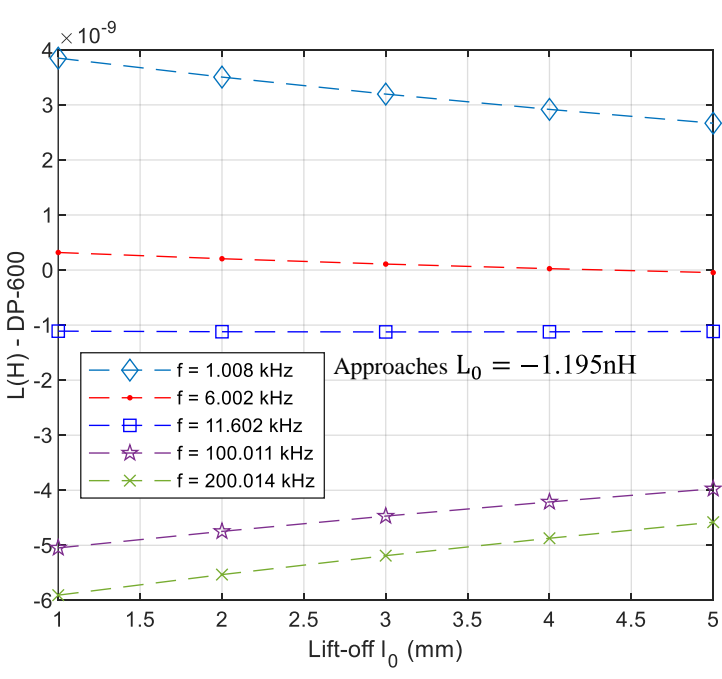

(a)

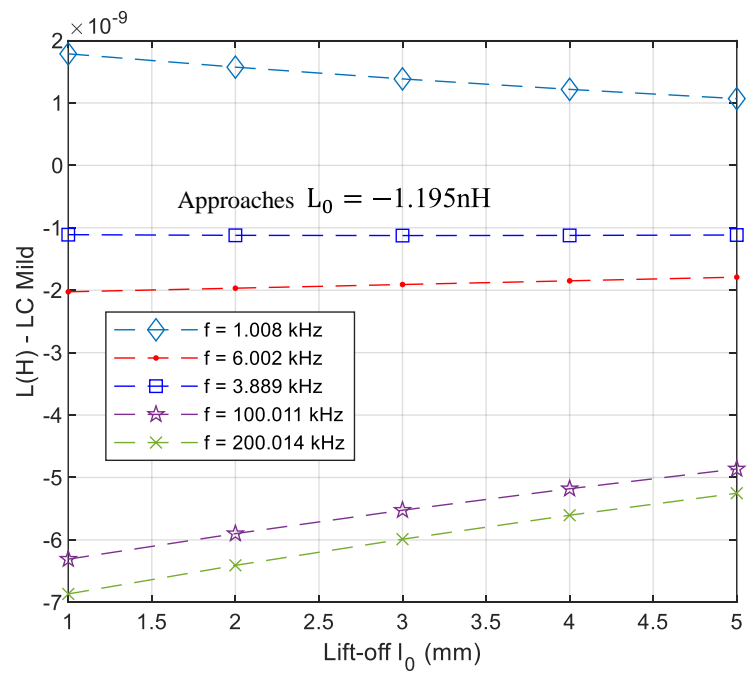

(b) 


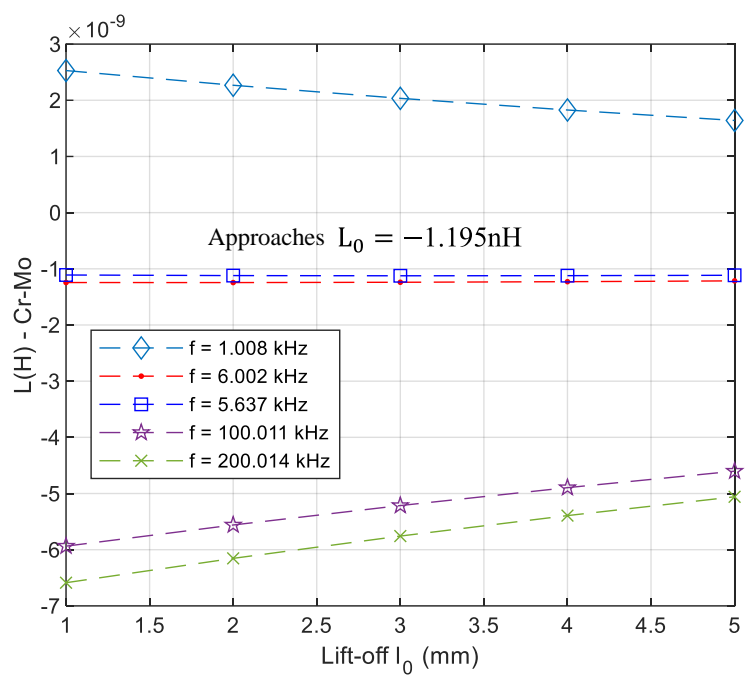

(c)

Fig. 6 Inductance change versus lift-offs of the sensor under a series of excitation frequencies

(a) DP-600: $\mathrm{L}\left(\mathrm{l}_{0}\right)$ approaches $\mathrm{L}_{0}$ around $11.602 \mathrm{kHz}$

(b) Low carbon Mild: $\mathrm{L}\left(\mathrm{l}_{0}\right)$ approaches $\mathrm{L}_{0}$ around $3.889 \mathrm{kHz}$

(c) Cr-Mo: $\mathrm{L}\left(\mathrm{l}_{0}\right)$ approaches $\mathrm{L}_{0}$ around $5.637 \mathrm{kHz}$

Fig. 6 illustrates the inductance change with different lift-off variations under a series of excitation frequencies for DP-600, low-carbon mild, and Cr-Mo steels. It can be observed that the inductance change of DP-600, lowcarbon mild, and Cr-Mo steels approaches $\mathrm{L}_{0}$, and becomes almost immune to the lift-off variation under the working frequency of $11.602,3.889$, and $5.637 \mathrm{kHz}$ (closest to LIF of each steel). Lower frequencies result in a larger inductance but (nearly linear) decrease with incremented lift-offs of the sensor. By increasing the frequency starting from the LIF, the magnitude of L increases, while, for a given frequency, it decreases as the $l_{0}$ increases. Moreover, as can be seen from the inductance change curve under 1.008, 6.002, 100.011, and 200.014 kHz, the inductance change gradually approaches the lift-off invariant inductance (LII) $-\mathrm{L}_{0}(-1.195 \mathrm{nH}$ for the sensor geometry listed in Table 1) and becomes more stable with increased lift-offs of the sensor when the working frequency is closer to the corresponding frequency of the intersected points - lift-off invariant frequency (LIF).

In Fig. 7, the relative error between the LII $\left(\mathrm{L}_{0}\right)$ and the inductance change (for DP-600, LC mild, and Cr-Mo steels) of the point that is closest to LII or LIF has been analysed under different lift-offs of the eddy current sensor. It can be found that three curves of the inductance change error for different steels follow the same trend and close values with the incremented lift-offs. With the increased lift-offs of the eddy current sensor, the inductance change closest to LII ( $\mathrm{L}$ in y axis of Fig.7) starts at a slightly lower value, then overshoots LII ( $\left.\mathrm{L}_{0}\right)$ and gradually reverses back to a lower value. Besides, the error of the inductance change is within $0.60 \%$ for a small range of lift-offs.

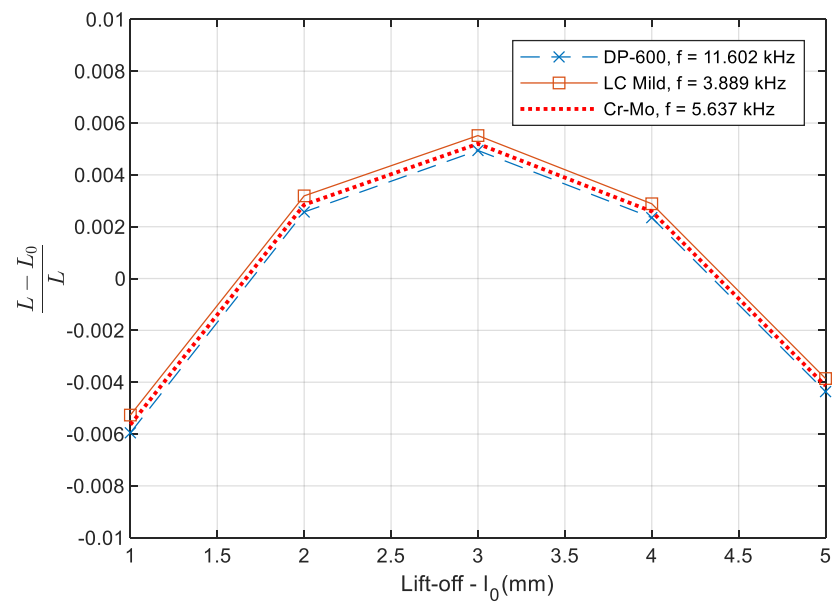

Fig. 7 Relative error of the inductance change (with respect to $\mathrm{L}_{0}$ ) under the frequencies at or closest to LII - $\mathrm{L}_{0}$ with different lift-offs of the sensor 


\section{Reconstruction of steel properties using lift-off invariant frequency (LIF)}

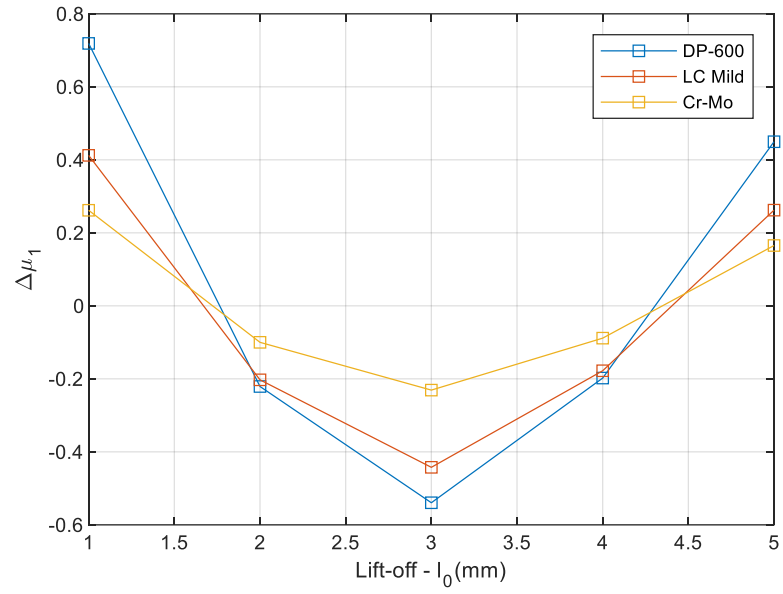

(a)

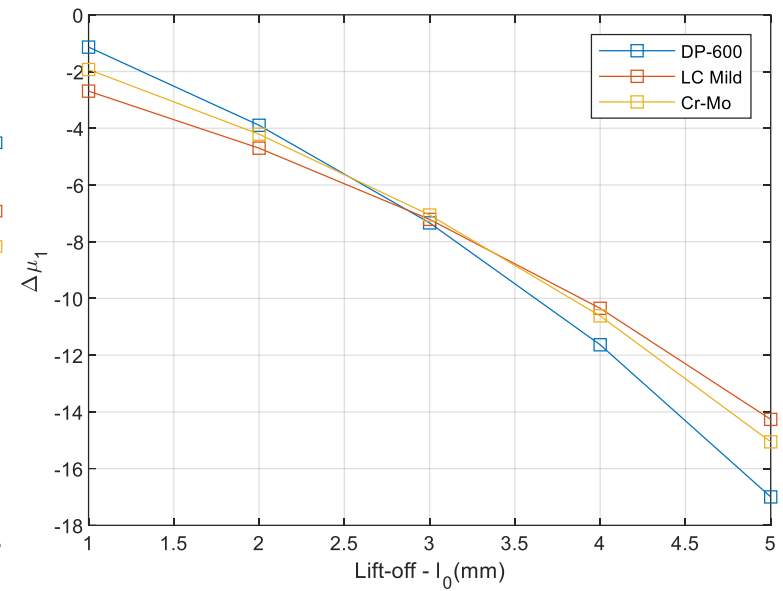

(b)

Fig. 8 Error of the reconstructed relative magnetic permeability with different lift-offs of the sensor (a) using the LIF and LII feature (b) using the zero-crossing feature [38] without considering the effect of lift-off parameter (i.e., assuming lift-off is zero) in the algorithm

Since the inductance change of the point that is closest to LII or LIF has been verified almost immune to a small range of lift-offs of the eddy current sensor, its value and the corresponding frequency (closest to LIF) can be further used to reconstruct one of the property of samples (either the electrical conductivity or the relative magnetic permeability; one must be known as a priori). The principle of the reconstruction is shown from (7) to (11), which is the modified Newton-Raphson iterative method. For example, to find the non-linear solution of the relative permeability $\mu_{1}$ of steels, parameters including the thickness (Table 1), electrical conductivity (Table 1), sensor geometry (Table 2), inductance change (closest to LII), and frequency (closest to LIF, as shown in the legend of Fig. 7) are inputted into equation (8) and (10). The relative permeability can be accurately derived when the difference of the inductance change between the current and last iterative loop $\left(\mathrm{L}\left(\mu_{1 \mathrm{r}}\right)-\mathrm{L}\left(\mu_{1 \mathrm{r}}^{\prime}\right)\right)$ converges at a small maximum interference threshold (MAXIT). As can be seen from Fig. 8 a, steels of DP-600, low-carbon mild, and Cr-Mo share a similar error trend of the reconstructed relative permeability. With the increased lift-off distance of the eddy current sensor, the reconstructed magnetic permeability begins with a higher value, then falls below the actual value and finally returns to a higher value. In Fig. 9 a, the reconstructed electrical conductivity of steels follows an inverse trend of the reconstructed permeability (but the similar trend of the inductance change shown in Fig. 7), which is due to the different sensitivities with respect to the inductance change. Overall, from Fig. 8 a, and Fig. 9 a, the properties of the steels can be reconstructed with a maximum relative error of $0.61 \%$ (with $0.32 \%$ for the reconstructed magnetic permeability, and $0.61 \%$ for the reconstructed electrical conductivity).

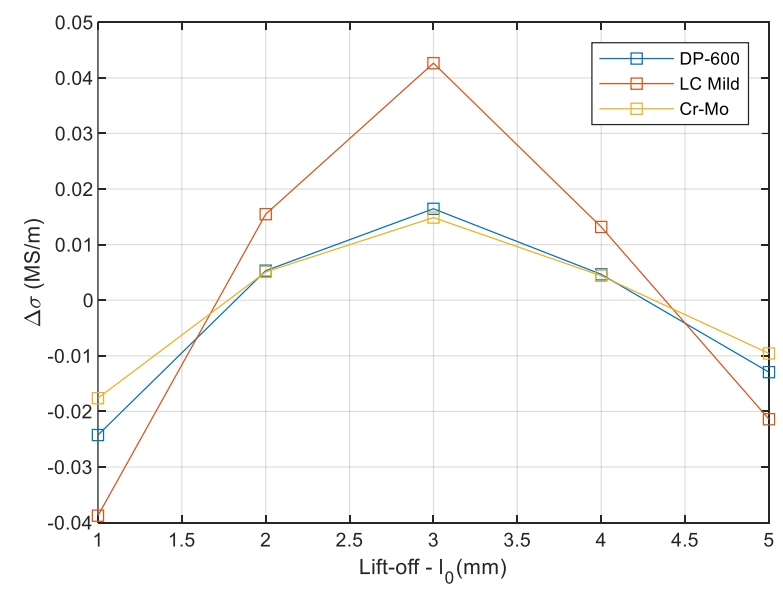

(a)

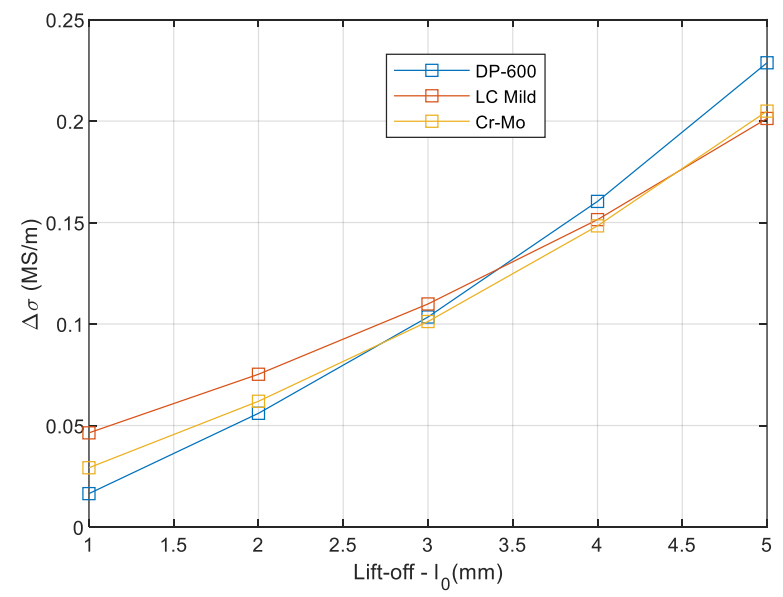

(b)

Fig. 9 Error of the reconstructed electrical conductivity with different lift-offs of the sensor (a) using the LIF and LII feature (b) using the zero-crossing feature [38] without considering the effect of lift-off parameter (i.e., assuming lift-off is zero) in the algorithm

Fig. 8 b, and Fig. 9 b show the retrieval of relative permeability and electrical conductivity using the zero- 
crossing feature (ZCF), which does not consider the effect of lift-off parameter (i.e., assuming lift-off is zero) in the algorithm. It can be seen that the retrieval error using the proposed LIF and LII feature is much lower than that using the ZCF feature, particularly for lift-offs over $2 \mathrm{~mm}$.

\section{Conclusions}

This paper presents the lift-off invariant inductance (LII) and corresponding lift-off invariant frequency (LIF) features in the multi-frequency eddy current testing. It has been found under the LIF (or the frequency sample closest to LIF), the measured inductance change (which is exactly the LII or significantly approaches LII) for the sensor above the test piece is almost immune to the lift-off distance. From the inductance measurement of three different types of steels, the LII is proofed only determined by the size of the sensor and barely affected by different steels. By inputting the known parameters (e.g., thickness and sensor geometries), LII, and LIF into the Newton-Raphson iterative inverse solver, one of the property of samples (either the electrical conductivity or the relative magnetic permeability; one must be known as a priori) is derived with a small error of $0.61 \%$ and 0.32 $\%$ respectively. Previously, the eddy current thin-skin regime [40] is proposed for the surface defect inspection, which does not consider the lift-off effect. In the future, more investigations will be carried out to test the feasibility of blending the proposed method (LIF feature) into the surface defect detection. Besides, inductance sensitivities to several parameters (electrical conductivity, magnetic permeability, thickness, and lift-off) have been analysed at different frequencies [34]. In the future, more tests will be conducted to optimise the sensor design for the augmentation of LIF sensitivities to a local change of electrical conductivity or magnetic permeability.

\section{Acknowledgments}

This work was supported by [UK Engineering and Physical Sciences Research Council (EPSRC)] [grant number: EP/P027237/1] [title: Real-time In-line Microstructural Engineering (RIME)].

Author Contributions: (Methodology, manuscript drafting) M. Lu and X. Meng; (conceptualization, manuscript revision) M. Lu and X. Meng; (experiment data curation, manuscript review) M. Lu, X. Meng, R. Huang, L. Chen; (Supervision) W. Yin, A. Peyton, M. Lu. All authors have read and agreed to the published version of the manuscript.

\section{References}

1. G. Yang, G. Dib, L. Udpa, A. Tamburrino and S. S. Udpa, "Rotating Field EC-GMR Sensor for Crack Detection at Fastener Site in Layered Structures," IEEE Sensors Journal, vol. 15, no. 1, pp. 463-470, Jan. 2015.

2. I. Z. Abidin, C. Mandache, G. Y. Tian and M. Morozov, "Pulsed eddy current testing with variable duty cycle on rivet joints," NDT \& E International, vol. 42, no. 7, pp. 599-605, 2009.

3. X. Chen and Y. Lei, "Electrical conductivity measurement of ferromagnetic metallic materials using pulsed eddy current method," NDT \& E International, vol. 75, pp. 33-38, 2015.

4. He, D. F., \& Yoshizawa, M. (2002). Dual-frequency eddy-current NDE based on high-Tc rf SQUID. Physica C: Superconductivity, 383(3), 223-226.

5. E. Pinotti and E. Puppin, "Simple Lock-In Technique for Thickness Measurement of Metallic Plates," IEEE Transactions on Instrumentation and Measurement, vol. 63, no. 2, pp. 479-484, Feb. 2014

6. C. C. Tai, J. H. Rose, and J. C. Moulder, "Thickness and conductivity of metallic layers from pulsed eddy-current measurements," Review of Scientific Instruments, vol. 67, no. 11, Aug. 1996.

7. D. Vasic, V. Bilas and D. Ambrus, "Pulsed eddy-current nondestructive testing of ferromagnetic tubes," IEEE Transactions on Instrumentation and Measurement, vol. 53, no. 4, pp. 1289-1294, Aug. 2004.

8. J. C. Moulder, E. Uzal, and J. H. Rose, "Thickness and conductivity of metallic layers from eddy current measurements," Review of Scientific Instruments, vol. 63, no. 6, 1992.

9. A. V. Egorov, V. V. Polyakov, D. S. Salita, E. A. Kolubaev, S. G. Psakhie, A. G. Chernyavskii, I. V. Vorobei, "Inspection of aluminum alloys by a multi-frequency eddy current method," Defence Technology, vol. 11, no. 2, pp. 99-103, 2015.

10. T. Theodoulidis and E. E. Kriezis, "Eddy current canonical problems (with applications to nondestructive evaluation)", Material Science, 2006.

11. R. Huang, M. Lu, A. Peyton, and W. Yin, "Thickness measurement of metallic plates with finite planar dimension using eddy current method," IEEE Transactions on Instrumentation and Measurement, early access, 2020. Doi:10.1109/TIM.2020.2987413.

12. Y. Shin, D. Choi, Y. Kim and S. Lee, "Signal characteristics of differential-pulsed eddy current sensors in the evaluation of plate thickness," NDT \& E International, vol. 42, no. 3, pp. 215-221, 2009.

13. W. Li, X. Yuan, G. Chen, J. Ge, X. Yin and K. Li, "High sensitivity rotating alternating current field measurement for arbitraryangle underwater cracks,” NDT\&E International, vol. 79, pp. 123-131, Apr. 2016. 
14. M. Lu, et al. "Acceleration of frequency sweeping in eddy-current computation," IEEE Transactions on Magnetics, vol. 53, pp. 18, 2017.

15. Y. He, G. Tian, H. Zhang, M. Alamin, A. Simm and P. Jackson, "Steel Corrosion Characterization Using Pulsed Eddy Current Systems," IEEE Sensors Journal, vol. 12, no. 6, pp. 2113-2120, 2012.

16. G. Tytko and L. Dziczkowski, "E-Cored Coil With a Circular Air Gap Inside the Core Column Used in Eddy Current Testing," IEEE Transactions on Magnetics, vol. 51, no. 9, pp. 1-4, Sept. 2015.

17. Ribeiro, A. L., Ramos, H. G., \& Arez, J. C. (2012). Liftoff insensitive thickness measurement of aluminum plates using harmonic eddy current excitation and a GMR sensor. Measurement, 45(9), 2246-2253.

18. B. A. Abu-Nabah, "Reduction of lift-off effect in high-frequency apparent eddy current conductivity spectroscopy," Measurement Science and Technology, vol. 28, no.5, pp. 055107, 2017

19. Kral, J., Smid, R., Ramos, H. M. G., \& Ribeiro, A. L. (2013). The lift-off effect in eddy currents on thickness modeling and measurement. IEEE Transactions on Instrumentation and Measurement, 62(7), 2043-2049.

20. S. Giguere, B. A. Lepine, and J. M. S. Dubois. "Pulsed eddy current technology: Characterizing material loss with gap and lift-off variations," Journal of Research in Nondestructive Evaluation, vol. 13, no. 3, pp. 119-129, 2001.

21. Y. Wang, M. Fan, B. Cao, B. Ye, and D. Wen, "Measurement of coating thickness using lift-off point of intersection features from pulsed eddy current signals," NDT \& E International, vol. 116, pp. 102333, 2020.

22. C.S. Angani et al. "Lift-off point of intersection feature in transient eddy-current oscillations method to detect thickness variation in stainless steel," IEEE Transactions on Magnetics, vol. 52, no. 6, pp. 1-8, 2016.

23. D. Wen, M. Fan, B. Cao, B. Ye and G. Tian, "Extraction of LOI Features from Spectral Pulsed Eddy Current Signals for Evaluation of Ferromagnetic Samples," IEEE Sensors Journal, vol. 19, no. 1, pp. 189-195, 1 Jan.1, 2019.

24. C. Mandache, and J. H. V. Lefebvre, "Transient and harmonic eddy currents: Lift-off point of intersection," NDT \& E International, vol. 39, no. 1, pp: 57-60, 2006

25. G. Y. Tian and A. Sophian, "Reduction of lift-off effects for pulsed eddy current NDT," NDT \& E International, vol. 38, no. 4, pp. 319-324, 2005.

26. W. Yin and A.J. Peyton, "Thickness measurement of non-magnetic plates using multi-frequency eddy current sensors," NDT \& E International, vol. 40, no. 1, pp. 43-48, 2007.

27. W. Yin et al, "Measurement of permeability and ferrite/austenite phase fraction using a multi-frequency electromagnetic sensor," NDT \& E International, vol. 42, no. 1, pp. 64-68, 2009.

28. M. Lu, H. Xu, W. Zhu, L. Yin et al. "Conductivity Lift-off Invariance and measurement of permeability for ferrite metallic plates," NDT \& E International, vol. 95, pp. 36-44, Apr. 2018.

29. W. Yin et al. "Permeability invariance phenomenon and measurement of electrical conductivity for ferrite metallic plates," InsightNon-Destructive Testing and Condition Monitoring, vol. 61, pp. 472-479, 2019.

30. M. Lu, W. Zhu, L. Yin, A. J. Peyton, W. Yin, and Z. Qu, "Reducing the lift-off effect on permeability measurement for magnetic plates from multifrequency induction data," IEEE Transactions on Instrumentation and Measurement, vol. 67, no. 1, pp. 167-174, Jan. 2018

31. M. Lu, L. Yin, A. J. Peyton and W. Yin, "A novel compensation algorithm for thickness measurement immune to lift-off variations using eddy current method," IEEE Transactions on Instrumentation and Measurement, vol. 65, no. 12, pp. 2773-2779, Dec. 2016.

32. M. Lu, R. Huang, W. Yin, Q. Zhao, and A. Peyton, "Measurement of permeability for ferrous metallic plates using a novel lift-off compensation technique on phase signature," IEEE Sensors Journal, vol. 19, no. 17, pp. 7440-7446, 1 Sept.1, 2019.

33. M. Lu et al. "Determining the magnetic permeability of ferrite steel strip by a custom inversion method," Proc. 12th ECNDT, pp. $1-8,2018$

34. M. Lu, et al. "Determination of the magnetic permeability, electrical conductivity, and thickness of ferrite metallic plates using a multi-frequency electromagnetic sensing system," IEEE Transactions on Industrial Informatics, vol. 15, pp. 4111-4119, 2019.

35. J.R.S. Avila, M. Lu et al. "Accurate measurements of plate thickness with variable lift-off using a combined inductive and capacitive sensor," NDT \& E International, vol. 110, pp. 102202, 2020.

36. M. Lu, R. Huang, W. Yin, Q. Zhao, and A. Peyton, "Measurement of permeability for ferrous metallic plates using a novel lift-off compensation technique on phase signature," IEEE Sensors Journal, vol. 19, no. 17, pp. 7440-7446, 1 Sept.1, 2019.

37. M. Lu, X. Meng, W. Yin, Z. Qu, F. Wu, J. Tang, et al., "Thickness measurement of non-magnetic steel plates using a novel planar triple-coil sensor," NDT \& E International, vol. 107, 2019.

38. M. Lu et al. "Measurement of ferromagnetic slabs permeability based on a novel planar triple-coil sensor," IEEE Sensors J., vol. 20, no. 6, pp. 2904-2910, 2020.

39. C. V. Dodd, and W. E. Deeds, “Analytical solutions to eddy-current probe-coil problems," Journal of applied physics, vol. 39, no. 6, pp. 2829-2838, 1968

40. M. Lu, et al, "Determination of surface crack orientation based on thin-skin regime using triple-coil drive-pickup eddy-current sensor," IEEE Transactions on Instrumentation and Measurement, vol. 70, 2020.

41. J.R.S. Avila, M. Lu et al. "Accurate measurements of plate thickness with variable lift-off using a combined inductive and capacitive sensor," NDT \& E International, vol. 110, pp. 102202, 2020.

42. M. Lu, L. Yin, A. J. Peyton and W. Yin, "A novel compensation algorithm for thickness measurement immune to lift-off variations using eddy current method," IEEE Transactions on Instrumentation and Measurement, vol. 65, no. 12, pp. 2773-2779, Dec. 2016.

43. M. Lu, X. Meng, W. Yin, Z. Qu, F. Wu, J. Tang, et al., "Thickness measurement of non-magnetic steel plates using a novel planar triple-coil sensor,” NDT \& E International, vol. 107, 2019.

44. R. Huang, M. Lu, A. Peyton, and W. Yin, "Thickness measurement of metallic plates with finite planar dimension using eddy current method," IEEE Transactions on Instrumentation and Measurement, early access, 2020. Doi:10.1109/TIM.2020.2987413.

45. M. Lu et al. "Measurement of ferromagnetic slabs permeability based on a novel planar triple-coil sensor," IEEE Sensors J., vol. 20, no. 6, pp. 2904-2910, 2020.

46. M. Lu, W. Zhu, L. Yin, A. J. Peyton, W. Yin, and Z. Qu, "Reducing the lift-off effect on permeability measurement for magnetic plates from multifrequency induction data," IEEE Transactions on Instrumentation and Measurement, vol. 67, no. 1, pp. 167-174, Jan. 2018

47. C. V. Dodd, and W. E. Deeds, “Analytical solutions to eddy-current probe-coil problems," Journal of applied physics, vol. 39, no. 6, pp. 2829-2838, 1968.

48. R. Huang, M. Lu et al, "Measurement of the radius of metallic plates based on a novel finite region eigenfunction expansion (FREE) method,” IEEE Sensors Journal, vol. 20, pp. 15099 - 15106, 2020. Doi: 10.1109/JSEN.2020.3009443. 
49. R. Huang, M. Lu et al, "Measuring co-axial hole size of finite-size metallic disk based on a dual-constraint integration feature using multi-frequency eddy current testing," IEEE Transactions on Instrumentation and Measurement, vol. 70, pp. 1-7, 2020. Doi: 10.1109/TIM.2020.3026762.

50. M. Lu et al, "Thickness Measurement of Metallic Film Based on a High-Frequency Feature of Triple-Coil Electromagnetic Eddy Current Sensor," IEEE Transactions on Instrumentation and Measurement, vol. 70, pp. 1-8, 2020. Doi: 10.1109/TIM.2020.3027929.

51. M. Lu et al, "Measuring Lift-Off Distance and Electromagnetic Property of Metal Using Dual-Frequency Linearity Feature," IEEE Transactions on Instrumentation and Measurement, vol. 70, pp. 1-9, 2020. Doi: 10.1109/TIM.2020.3029348.

52. M. Lu et al, "Lift-off tolerant pancake eddy-current sensor for the thickness and spacing measurement of non-magnetic plates," IEEE Transactions on Instrumentation and Measurement, early access, 2020. Doi: 10.1109/TIM.2020.3033377.

53. Chew, W. C. Waves and Fields in Inhomogenous Media. New York, NY, USA: IEEE Press, 1995, chapter. 2, pp. 4-10.

54. M. Lu et al, "Inversion of distance and magnetic permeability based on material-independent and lift-off insensitive algorithms using eddy current sensor," IEEE Transactions on Instrumentation and Measurement, early access, 2020. Doi: 10.1109/TIM.2020.3036099.

55. W. Yin et al, "Measurements of Thickness for Metallic Plates With Co-Axial Holes Using a Novel Analytical Method With the Modified Integration Range," IEEE Access, vol. 8, pp. 198301 - 198306, 2020.

56. X. Meng, M. Lu et al, "Inversion of lift-off distance and thickness for non-magnetic metal using eddy current testing," IEEE Transactions on Instrumentation and Measurement, vol. 70, 2020. Doi: 10.1109/TIM.2020.3038289.

57. M. Lu, X. Meng, et al, "Determination of surface crack orientation based on thin-skin regime using triple-coil drive-pickup eddycurrent sensor," IEEE Transactions on Instrumentation and Measurement, early access, 2020. Doi: 10.1109/TIM.2020.3044729.

58. Yin, W.; Tang, J.; Lu, M.; et al. An equivalent-effect phenomenon in eddy current non-destructive testing of thin structures. IEEE Access, 2019, 7, pp. 70296-70307.

59. Lu, M.; et al. Determination of Surface Crack Orientation Based on Thin-Skin Regime Using Triple-Coil Drive-Pickup EddyCurrent Sensor. IEEE Transactions on Instrumentation and Measurement, 2020, 70, pp. 1-9. DOI: 10.1109/TIM.2020.3044729

60. M. Lu, et al., "Prediction of the asymptotical magnetic polarization tensors for cylindrical samples using the boundary element method," In 2015 IEEE Sensors Applications Symposium (SAS), pp. 1-4. IEEE, 2015.

61. R. Huang, M. Lu, A. Peyton, and W. Yin, "A novel perturbed matrix inversion based method for the acceleration of finite element analysis in crack-scanning eddy current NDT," IEEE Access, vol. 8, pp. 12438-12444, 2020.

62. J. Tang et al., "A Novel Efficient FEM Thin Shell Model for Bio-Impedance Analysis," Biosensors, vol. 10, no. 6, pp. 69, 2020.

63. L. Chen, et al., "Textile Based Capacitive Sensor for Physical Rehabilitation via Surface Topological Modification," ACS Nano, vol. 14, no. 7, pp. 8191-8201, 2020. DOI: 10.1021/acsnano.0c01643

64. Z. Jin, et al., "Methods of Controlling Lift-off in Conductivity Invariance Phenomenon for Eddy Current Testing," IEEE ACCESS, vol. 8, pp. 2169-3536, 2020. DOI: 10.1109/ACCESS.2020.3007216.

65. J. Tang, et al., "Effect of frozen-thaw injury on cell membrane and bio-impedance," In 2020 IEEE International Instrumentation and Measurement Technology Conference (I2MTC), pp. 1-6. IEEE, 2020.

66. J. Tang, et al., "Bio-impedance spectroscopy for frozen-thaw of bio-samples: Non-contact inductive measurement and finite element (FE) based cell modelling," Journal of Food Engineering, vol. 272, pp. 109784, 2020.

67. H. Xu et al., "Imaging a weld cross-section using a novel frequency feature in multi-frequency eddy current testing," InsightNon-Destructive Testing and Condition Monitoring, vol. 61, no. 12, pp. 738 - 743, 2019.

68. Y. Xie et al., "Novel Wearable Sensors for Biomechanical Movement Monitoring Based on Electromagnetic Sensing Techniques," IEEE Sensors Journal, vol. 20, no. 2, 2020. DOI: 10.1109/JSEN.2019.2943487

69. W. Yin et al., "Permeability invariance phenomenon and measurement of electrical conductivity for ferrite metallic plates," Insight-Non-Destructive Testing and Condition Monitoring, vol. 61, no. 8, pp. 472 - 479, 2019.

70. M. Lu et al., "A model for the triboelectric nanogenerator with inductive load and its energy boost potential," Nano Energy, vol. 63, pp. 103883, 2019.

71. M. Lu et al., "Forward solver for deep earth exploration and induction logging using custom built Edge-Element FEM technique," Acta Geologica Sinica, vol. 93, pp. 302-304, 2019.

72. L. Chen et al., "Whole System Design of Wearable Magnetic Induction Sensor for Physical Rehabilitation," Advanced Intelligent Systems, vol. 1, no. 1, pp. 1900037, 2019.

73. Y. X et al., "A self-powered radio frequency (RF) transmission system based on the combination of triboelectric nanogenerator (TENG) and piezoelectric element for disaster rescue/relief," Nano Energy, vol. 54, pp. 331-340, 2018.

74. W. Yin et al., "Custom edge-element FEM solver and its application to eddy-current simulation of realistic 2M-element human brain phantom," Bioelectromagnetics, vol. 39, no. 8, pp. 604-616, 2018.

75. L. Yin et al., "Detection of corrosion pits based on an analytically optimised eddy current sensor," Insight-Non-Destructive Testing and Condition Monitoring, vol. 60, no. 10, pp. 561-567, 2018.

76. W. Yin et al., "Acceleration of eddy current computation for scanning probes," Insight-Non-Destructive Testing and Condition Monitoring, vol. 60, no. 10, pp. 547-555, 2018.

77. W. Zhou et al., "Three-dimensional electromagnetic mixing models for dual-phase steel microstructures," Applied Sciences, vol. 8 , no. 4, pp. 547-555, 2018.

78. M. Lu, et al., "Determining the magnetic permeability of ferrite steel strip by a custom inversion method," In Proc. 12th ECNDT, pp. 1-8. 2018.

79. J. Tang, et al., "Cellular structure analysis based on magnetic induction finite element method simulations and measurements," bioRxiv, pp. 275271, 2018. DOI: 10.1101/275271

80. J.R.S. Avila, et al., "A novel dual modality sensor with sensitivities to permittivity, conductivity, and permeability," IEEE Sensors Journal, vol. 18, no. 1, pp. 356-362, 2017.

81. T. Yang, et al., "Level measurement for saline with a small surface area using high frequency electromagnetic sensing technique," Measurement, vol. 101, pp. 118-125, 2017.

82. X. Meng, et al, "Evaluation of coating thickness using lift-off insensitivity of eddy current sensor," Sensors, vol. 21, no. 2, pp. $419,2021$.

83. X. Meng, et al, "Evaluation of coating thickness using lift-off insensitivity of eddy current sensor," Sensors, vol. 21, no. 2, pp. 419, 2021.

84. M. Lu, et al., " Thickness measurement of circular metallic film using single-frequency eddy current sensor," NDT \& E International, vol. 119, pp. 102420, 2021. 